\title{
ESTRATEGIAS INTERNACIONALES DE CONSERVACIÓN IMPLEMENTADAS EN EL SISTEMA NACIONAL DE ÁREAS PROTEGIDAS EN COLOMBIA
}

\author{
INTERNATIONAL CONSERVATION STRATEGIES \\ IMPLEMENTED IN THE NATIONAL SYSTEM OF PROTECTED \\ AREAS IN COLOMBIA
}

Paulina Alejandra Vergara Buitrago

Ingeniera Ambiental, Especialista en Gestión Ambiental, Ms(c) Geografía

Universidad pedagógica y Tecnológica de Colombia - Grupo Sistemática Biológica (SisBio)

paulina.vergara@uptc.edu.co

\begin{abstract}
Resumen
Colombia cuenta con 1044 áreas naturales protegidas, las cuales constituyen la principal herramienta para la conservación de la biodiversidad y sus servicios ecosistémicos. Razón por la cual, la ejecución de estrategias complementarias para la conservación de la diversidad biológica en las áreas naturales, constituye un mecanismo para garantizar la funcionalidad de los ecosistemas colombianos. En el presente artículo se hizo una revisión en torno al establecimiento de las estrategias internacionales denominadas como sitios RAMSAR, Áreas importantes para la Conservación de Aves, Reservas para Aves Playeras, Reservas de la Biósfera y sitios de Patrimonio Natural que han sido reconocidos al interior de las áreas naturales protegidas de Colombia, esto con el propósito de identificar y aportar información sobre el estado actual de implementación de estas estrategias
\end{abstract}

de conservación en el Sistema Nacional de Áreas Protegidas de Colombia.

Palabras clave: conservación ambiental; desarrollo; ecosistema; gestión; recursos naturales.

\begin{abstract}
Colombia has 1044 protected natural areas, which are the main tool for the conservation of biodiversity and its ecosystem services. Therefore, the implementation of complementary strategies for the conservation of biological diversity in natural areas is a mechanism to guarantee the functionality of Colombian ecosystems. In the present article, a review was made about the establishment of international strategies known as RAMSAR sites, important areas for Bird Conservation, Shorebird Reserves, Biosphere Reserves and Natural Heritage Sites that have been
\end{abstract}


recognized as interior of the protected natural areas of Colombia, this with the purpose of identifying and providing information on the current state of implementation of these conservation strategies in the National System of
Protected Areas of Colombia.

Keywords: development; ecosystem; environmental protection; management; natural resources.

\section{Resumen gráfico}

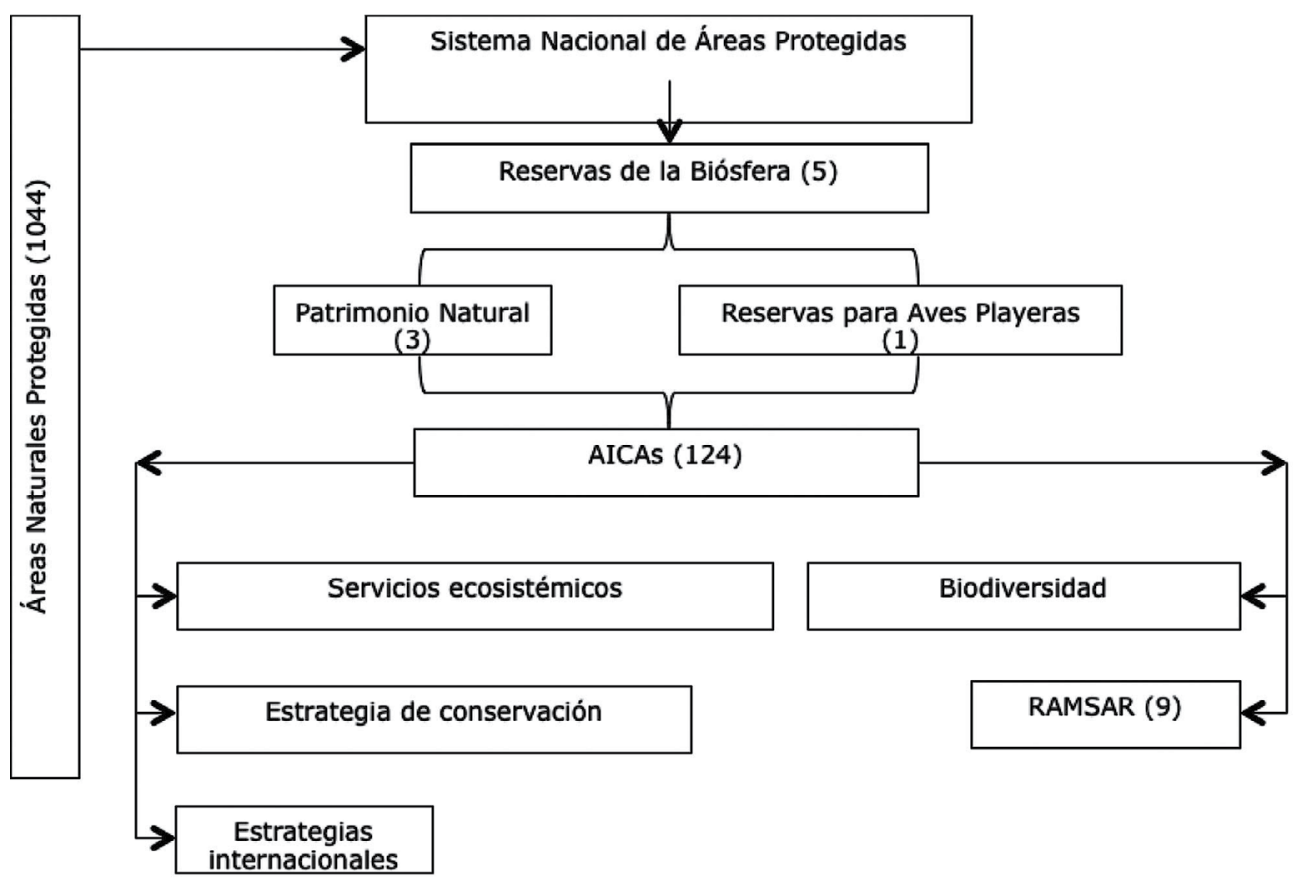

\section{Introducción}

Colombia es considerada como uno de los países megadiversos del mundo (Andrade, 2011; Arbeláez, 2013; Mongabay, 2016), condición que se ve reflejada en la amplia variedad de ecosistemas presentes en el territorio, desde páramos hasta bosques andinos, selvas húmedas tropicales, bosques secos, humedales, sabanas y zonas áridas (Márquez, 2001; IDEAM et al., 2007, Romero et al., 2008).

La importancia de la biodiversidad, así como la adopción de medidas para su conservación, uso sostenible y distribución de beneficios que se deriven de su utilización, se consignan en la Convención sobre Diversidad Biológica, ratificada por Colombia en la Ley
165 de 1994 (Ministerio de Medio Ambiente, 1996; MADS, 2012).

La estrategia de conservación in-situ del Sistema de Nacional de Áreas Protegidas (SINAP) incluye la reducción de los procesos y actividades que causan pérdida o deterioro de la biodiversidad y la recuperación de ecosistemas degradados y de especies amenazadas (Hurtado et al., 2013; De la maza et al., 2003).

El reto de la conservación en áreas naturales protegidas consiste en enfocar la gestión a la planificación y manejo de la matriz de paisaje circundante (Borrini et al., 2014), buscando oportunidades y estrategias que contribuyan a 
mantener los procesos ecológicos de los ecosistemas como objetos de conservación (Vargas, 2011), incluyendo la conectividad entre los actores, las estructuras socioeconómicas y las instituciones relacionadas (Hansen y DeFries 2007; Chaves y Hurtado 2007).

Así mismo, existen otros convenios y programas internacionales, que aplicados a las áreas naturales protegidas han permitido reforzar su conservación (Rodríguez, 2009; Ávila y Hasmine, 2015), ya que otorgan un reconocimiento internacional, que trasciende fronteras y constituye una poderosa herramienta para elevar su importancia y adquirir mayor prestigio y visibilidad mundial, al conjuntar intereses y voluntades en torno a la conservación de estas áreas y su biodiversidad (Orellana, 2014; López y Vidargas, 2016).

La representatividad de las áreas protegidas de Colombia se ha complementado con estrategias de conservación internacionales con denominaciones como: Sitios Ramsar, Áreas Importantes para la Conservación de Aves, Red Hemisférica de Reservas para Aves Playeras, Reservas de la Biósfera o sitios de Patrimonio de la Humanidad (Decreto 2372, 2010; Conpes 3680, 2010; Hernández, 2011).

Este artículo, por todo lo anterior, describió e identificó el número de estrategias internacionales de conservación (Humedales Ramsar, Áreas importantes para la Conservación de Aves, Reservas para Aves Playeras, Reservas de la Biósfera y sitios de Patrimonio de la Humanidad) articulas actualmente con el SINAP, esto con el fin de reconocer las áreas naturales que han adoptado estrategias internacionales.

\section{Metodología}

Se realizó una revisión de información disponible en Parques Nacionales Naturales de Colombia, la Secretaria de la Convención de
Ramsar, la BirdLife International, la Red Hemisférica de Reservas para Aves Playeras, la Organización de las Naciones Unidas para la Educación, la Ciencia y la Cultura; así como, los estudios de los Institutos de Investigación vinculados al Ministerio de Ambiente y Desarrollo Sostenible, el Instituto de Investigación de Recursos Biológicos Alexander von Humboldt, el Instituto de Hidrología, Meteorología y la Asociación Calidris.

Del Registro Único Nacional de Áreas Protegidas (RUNAP) se descargaron los shapes de las áreas protegidas de Colombia, y del Sistema de Información Ambiental para Colombia (SIAC) los shapes de las estrategias internacionales. A partir de la información de las entidades geográficas cartografiables a escala 1:100.000, se identificó el número estrategias internacionales en jurisdicción de áreas naturales protegidas; así como, el área total de implementación de estas áreas en el territorio colombiano, esta actividad se desarrolló por medio de un análisis visual en pantalla en el software ArcGis versión 10.5.

\section{Humedales Ramsar}

La convención sobre los humedales es un tratado intergubernamental aprobado el 2 de febrero de 1971 en la localidad iraní de Ramsar, situada a orillas del mar Caspio (Schnack, 2001; Valencia, 2004; Romero, 2013), se considera el primer tratado moderno sobre conservación y uso sostenible de los recursos naturales (Secretaría de la Convención de Ramsar, 2016).

Los humedales son reconocidos a nivel mundial como ecosistemas estratégicos que generan servicios ecosistémicos (Castellanos, 2006), como son la depuración del agua, la exportación de nutrientes, el control de inundaciones, el control de erosión, almacenamiento de agua, la regulación hidrológica, provisión de 
hábitat para especies, entre otros (MEA, 2005; Ricaurte, 2014), los cuales impactan de forma directa en el bienestar humano de las poblaciones que viven en estrecha relación con los mismos, pero también de manera indirecta a una escala mundial (De Groot et al., 2006).

Asimismo, se consideran un elemento vital dentro del amplio mosaico de ecosistemas con que cuenta el Colombia y se constituyen por su oferta de servicios ecosistémicos en un renglón importante de la economía nacional, regional y local (Andrade et al., 2002). A través de la Ley 357 del 21 de enero 1997, Colombia incorporó a la legislación nacional este tratado con origen en el derecho público internacional, adhesión que fue ratificada en junio de 1998 (Arango et al., 2008).

La normativa colombiana reconoce la protección de los humedales como un asunto vital para alcanzar los propósitos generales de conservación de la biodiversidad previstos en tratados internacionales (Pastrana y Pacheco, 2010), como es el Convenio Internacional sobre Diversidad Biológica y las obligaciones relacionadas que se derivan de los tratados (Alcaldía Mayor de Bogotá, 2006).

Colombia ha adoptado una posición de vanguardia a nivel mundial en cuanto a la temática de los humedales, encaminándose no sólo a realizar un simple inventario nacional de estos ecosistemas, sino exigiendo además a las autoridades ambientales el deber de delimitarlos, con el fin de mejorar la gestión de su biodiversidad y de los servicios ecosistémicos de los cuales las sociedades dependen (Garzón y Gutiérrez, 2013).

En la actualidad hay declarados 12 sitios Ramsar en Colombia, con una superficie total de 1.871.802 ha, de los cuales 8 tienen jurisdicción en áreas naturales protegidas (Ver anexo
Tabla 1). Dichos sitios propenden por la conservación y el uso racional de los humedales, mediante el mantenimiento de sus características ecológicas y la implementación de enfoques de desarrollo sostenible (SIAC, 2018). El país con mayor sitios Ramsar es el Reino Unido (174) con una superficie de 1.281.989 ha (Ramsar, 2018).

Áreas importantes para la conservación de aves (AICAs)

El programa AICAs se originó a partir de una serie de estudios inéditos que a principios de los años ochenta realizaron la BirdLife International y la Oficina Internacional para la Investigación de los Humedales, por encargo de la Comisión de la Comunidad Europea y el Consejo de Europa (CCA, 1999).

Lo anterior con el objetivo de identificar, proteger y conseguir una gestión adecuada de una red de espacios prioritarios para la supervivencia de las poblaciones de aves a lo largo y ancho del mundo (Fundación ProAves, 2014). Este programa lo desarrollan en sus respectivos países todos los socios de BirdLife International intentando conseguir una protección legal, una gestión y un seguimiento de estos lugares (Infante et al., 2011).

La postulación de AICAs consiste en la identificación de un sitio que cumpla por lo menos con alguna de las siguientes circunstancias: 1) Mantener un número significativo de una o más especies amenazadas a nivel mundial, 2) Hacer parte de centros geográficos que contengan especies de rango restringido o restringidas a un bioma y 3) Contar excepcionalmente con un gran número de especies migratorias o gregarias (BirdLife, 2018a).

En Colombia las AICAs comenzaron a mediados del 2001 con el objetivo de crear una red 
nacional de conservación para las aves (Renjifo et al., 2002). Para lo cual, se han implementado estándares y métodos propuestos por la BirdLife International en políticas sobre conservación de biodiversidad (Franco et al., 2012). Así mismo, se han desarrollado también criterios nacionales tomando en cuenta la Lista Roja de aves nacional (Renjifo et al., 2000).

Con 1.921 especies de aves, Colombia es conocida como el país con la mayor riqueza y diversidad de aves del mundo (Fundación Proaves, 2015). Esta gran diversidad además de ser un privilegio es una gran responsabilidad, pues constituye un patrimonio universal que debe ser manejado para garantizar su preservación a perpetuidad (Katta, 2005).

La conservación de la biodiversidad en términos económicos y éticos, es un objetivo central en los convenios multilaterales de organizaciones gubernamentales y no gubernamentales alrededor del mundo (Renjifo et al., 2000). De acuerdo a la BirdLife (2018b), en Colombia se han identificado 124 AICAs (Tabla 2, Anexo), las cuales representan 8.095.371 ha, el $7,1 \%$ de la superficie nacional. De las 124 AICAs 88 se encuentran incluidas en áreas naturales del SINAP. El país con más AICAS es Estados Uni-dos (644) con una superficie de 117.730.000 ha (BirdLife, 2018c).

\section{La Red Hemisférica de Reservas para Aves Playeras (RHRAP)}

La RHRAP fue creada en 1986 en respuesta a la alarmante disminución de las poblaciones de varias especies de aves playeras de las Américas, y con el objetivo de identificar y proteger los sitios críticos utilizados por estas aves durante la migración anual (Blanco, 1999).

La RHRAP trabaja junto a gobiernos e instituciones privadas para proteger a millones de aves playeras migratorias y sus hábitats en las
Américas, brindando reconocimiento internacional a los sitios críticos utilizados por estas aves y promoviendo su manejo cooperativo y protección a nivel local (Pulido, 2017).

Las metas y objetivos del programa de la RHRAP se han articulado con el de otras estrategias internacionales como: la Convención Ramsar, la Convención sobre Especies Migratorias, el Convenio sobre la Diversidad Biológica, la Iniciativa de Especies Migratorias del Hemisferio Occidental, el Programa de Áreas Importantes para las Aves de BirdLife International y el programa del Censo de Aves Acuáticas Neotropicales de Wetlands International (Castillo et al., 2011).

Colombia cuenta con un área reconocida en la RHRAP, el delta del Río Iscuandé (DRI), con 4.000 ha se ubica en la costa Pacífica noroccidental de Colombia, departamento de Nariño, en límites con el departamento del Cauca (WHSRN, 2018), sin embargo esta área no está articula con ninguna área del SINAP. La avifauna de la DRI está compuesta por 53 especies de aves acuáticas y 45 especies de aves terrestres para un total de 98 especies de aves agrupadas en 18 órdenes y 37 familias (Asociación Calidris, 2011).

De los sitios importantes para aves playeras (prioritarios y potenciales), 34 se encuentran identificados en el directorio de AICAs Colombia (Asociación Calidris, 2009). Los litorales Caribe y Pacífico con 1.937 km y 1.536 km de línea costera, respectivamente (Invemar, 2015) acogen el 86 \% de aves playeras en Colombia (Asociación Calidris, 2010). El país con más áreas para la protección de aves playeras es Estados Unidos con 49 (WHSRN, 2018).

\section{Reservas de la Biósfera}

Las Reservas de la Biósfera (RB) son una iniciativa del programa sobre el Hombre y la 
Biósfera de la UNESCO que busca armonizar los conceptos de conservación y desarrollo, a través de la relación equilibrada entre los humanos y la biósfera (García, 2003).

A diferencia de otros tipos de áreas de conservación in situ, las RB corresponden a un concepto creado, discutido y modificado por científicos, con el propósito de crear una alternativa distinta, pero no excluyente, a los parques nacionales y similares (Halffter, 2011).

La puesta en marcha de las RB es un desafío en el que se deben contemplar los contextos jurídicos, institucionales y administrativos del territorio (Gómez, 2011). A diferencia de las áreas protegidas que tienen objetivos más convencionales, las RB implican articular objetivos de gestión, formas de protección e intereses (Bioret, 2001).

Colombia cuenta con 5 RB que presentan 34.381.950 ha (Tabla 3, Anexo). Dichas reser-vas son protegidas por el Estado y por la Red Mundial de Biósferas, cuya función principal es la conservación de la biodiversidad del plane-ta y la utilización sostenible del territorio, son laboratorios en donde se estudia la gestión integrada de las tierras, del agua y de la biodi-versidad (Araya y Clüsener, 2010).

\section{Patrimonio natural}

Según, la UNESCO (1972), el patrimonio na-tural está constituido por las formaciones físi-cas, biológicas, geológicas, fisiográficas y las zonas estrictamente delimitadas que consti-tuyan el hábitat de especies animales y vege-tales amenazadas, que tengan un (valor uni-versal excepcional) desde el punto de vista estético o científico.

El patrimonio natural de Colombia está representado en 3 Áreas Naturales Protegías, en 1973 y declarado patrimonio en 1994, el Santuario de Fauna y Flora de la Isla Malpelo creado en 1995 y declarado patrimonio en 2006 (PNN, 2018) y el Parque Nacional Natural Serranía de Chiribiquete reservado y alinderado el 1989 y declarado patrimonio en 2018 (UNESCO, 2018) (Tabla 4), en estas áreas se protege gran parte del tesoro natural de Colombia; que es, al mismo tiempo, riqueza irremplazable para todo el planeta (Saldarriaga et al., 2011).

Tabla 4. Patrimonio Natural de Colombia

\begin{tabular}{l|c|c} 
Nombre & \multicolumn{1}{c}{$\begin{array}{c}\text { Fecha } \\
\text { designación }\end{array}$} & Área (ha) \\
\hline $\begin{array}{l}\text { Parque Nacional Natural } \\
\text { Los Katíos }\end{array}$ & 1994 & 72.000 \\
\hline $\begin{array}{l}\text { Santuario de Fauna y } \\
\text { Flora de la Isla Malpelo }\end{array}$ & 2006 & 857.500 \\
\hline $\begin{array}{l}\text { Parque Nacional Natural } \\
\text { Serranía de Chiribiquete }\end{array}$ & 2018 & 4.268 .095 \\
\hline & Total & 5.197 .595 \\
\hline
\end{tabular}

Fuente de consulta: adaptado de: UNESCO (2018).

El Parque Katíos tiene su origen en lo que fue la hacienda Sautatá (que en lengua Kuna quiere decir metal que brilla) y en los asentamientos aledaños como Bijao y Travesía (Duarte et al., 2012). El parque se divide en tres sectores: montañas y colinas, planicies sin inundaciones y pantanos. La zona protegida hace parte del Tapón del Darién, uno de los lugares del mundo con mayor riqueza hídrica (Ministerio de Cultura, 2011).

La importancia del Parque estriba en la posibilidad de conservar importantes refugios de bosque y mantener la continuidad del corredor biológico entre el Centro y Suramérica, es decir, un puente de intercambio de biotas (Díaz et al., 2006). En este Parque es posible comprobar por que Colombia es uno de los países con mayor diversidad biológica, pues alrededor 
del $20 \%$ de las especies animales del país se encuentran allí (Puerta et al., 2013).

El Santuario de Malpelo conforma una de las cinco ecorregiones naturales de Colombia, conteniendo un mosaico de ecosistemas terrestres, litorales y submareales únicos que albergan especies terrestres y marinas endémicas (Díaz y Gómez, 2000; PNN, 2015). Malpelo alberga las más importantes formaciones coralinas del Pacífico colombiano la roca viviente de 376 metros de altura, se encuentra escoltada por un conjunto de once peñascos que sobresalen 10 metros sobre la superficie marina (Cano y Díaz, 2002).

Su posición geográfica y constitución geológica hacen de Malpelo un lugar excepcional para la agregación de una gran variedad de fauna marina, incluyendo varias especies pelágicas como tiburones, mantas, tortugas, atunes y delfines (Herrón et al., 2007; García, 2010). Además, constituye junto con las islas Galápagos, de Coco, de Coiba y Gorgona el corredor marino de conservación del Pacífico Oriental Tropical (POT) (Rodríguez y Giraldo, 2011).

El Parque Nacional Natural Serranía del Chiribiquete es una de las áreas más apartadas e inhóspitas del país, pero al mismo tiempo representa una valiosa riqueza natural y cultural. Y quizás uno de esos lugares de la tierra, donde el hombre adquirió mecanismos especiales de convivencia con su entorno, un profundo respeto al culto de los animales selváticos y al equilibrio perfecto entre el hombre y la naturaleza (Castaño, 1999).

\section{Conclusiones}

De las 1044 áreas naturales de Colombia, 25 presentan jurisdicción en humedales con categoría Ramsar, 174 están en Áreas Importantes para la Conservación de Aves, 190 se encuentran en Reservas de la Biosfera y 3 se ubican en áreas de Patrimonio Natural, en las reservas de aves playeras no se presenta áreas. Se recomienda continuar con la implementación de estrategias de conservación internacionales, con el fin de reforzar los procesos de gestión y visibilización de las áreas naturales protegidas de Colombia.

En Colombia hay 1 RHRAP, 4 sitios Ramsar y 88 AICAs, sin articulación al SINAP. Las cuales deben ser reconocidas, con el fin de diseñar una zonificación ambiental que promueva la planificación y desarrollo sostenibles de las áreas.

Las estrategias internacionales de sitios RAMSAR, Áreas importantes para la Conservación de Aves, Reservas para Aves Playeras, Reservas de la Biósfera y sitios de Patrimonio Natural, complementan los objetivos nacionales de conservación en Colombia, enfocados en asegurar la continuidad de los procesos ecológicos y evolutivos naturales que soportan la diversidad biológica de los ecosistemas y el suministro de servicios ecosistémicos esenciales para el bienestar humano y la permanencia del medio natural o de alguno de sus componentes.

\section{Agradecimientos}

A la Alianza del Pacífico en su plataforma de movilidad estudiantil y académica, que permitió realizar una estancia investigativa en la Universidad Nacional Mayor de San Marcos (Lima, Perú). Al Departamento Administrativo de Ciencia, Tecnología e Innovación COLCIENCIAS, a través de la Beca-pasantía Jóvenes Investigadores e Innovadores por la paz 2017 y a la Universidad Pedagógica y Tecnológica de Colombia.

\section{Literatura citada}

Alcaldía Mayor de Bogotá. (2006). Política de humedales del distrito capital. Departamento Administrativo del Medio Ambiente-DAMA. Bogotá D.C., Colombia. 14 p. 
Andrade, A., Rivera, M., Caicedo, D., \& Camargo, L. (2002). Política Nacional para humedales interiores de Colombia. Estrategias para su conservación y uso racional. Ministerio del Medio Ambiente. Ministerio del Medio Ambiente, Consejo Nacional Ambiental. 6 p.

Andrade, M. (2011). Estado del conocimiento de la biodiversidad en Colombia y sus amenazas. Consideraciones para fortalecer la interacción ciencia-política. Revista de la Academia Colombiana de Ciencias Exactas, Físicas y Naturales, 35(137), 491-507.

Arango, M., Lenis, M., \& Ramírez, N. (2008). Análisis sobre la aplicabilidad de las herramientas de gestión ambiental para el manejo de los humedales naturales interiores de Colombia. Gestión $y$ ambiente, 11(2).

Araya, P., \& Clüsener, M. (2010). Reservas de la biosfera. Su contribucion a la provision de servicios de los ecosistemas. Experiencias exitosas en Iberoamerica. Chile: Ed. Valent. 88 p.

Arbeláez, E. (2013). Describiendo especies: Un panorama de la biodiversidad Colombiana en el ámbito mundial. Acta Biológica Colombiana, 18(1), $165-178$.

Asociación Calidris. (2009). Sitios importantes para la conservación de las aves playeras en Colombia. Recuperado de: http://calidris.org.co/wpcontent/uploads/2009/09/Sitios-Importantesconservacion-playeras.pdf

Asociación Calidris. (2010). Plan de conservación para aves playeras en Colombia. Recuperado de: http://calidris.org.co/wp-content/ uploads/2010/10/plan_aves_playeras_colombia. pdf

Asociación Calidris. (2011). La bocana del Río Iscuandé (Nariño) diagnóstico biológico y socioeconómico. Fondo por Nuestros Mares. Recuperado de: https://docplayer.es/63053384-La-bocanadel-rio-iscuande-narino-diagnostico-biologico-ysocioeconomico.html

Bioret, F. (2001). Biosphere Reserve manager or coordinator?. Parks, 11 (1), 26-29.

BirdLife. (2018a). Data Zone. Recuperado de: http://datazone.BirdLife.org/country

BirdLife. (2018b). BirdLife International. Country profile: Colombia. Recuperado de: http://www. BirdLife.org/ datazone/country/Colombia
BirdLife. (2018c). BirdLife International. Country profile: USA. Recuperado de: http://datazone. birdlife.org/country/usa

Blanco, D. (1999). Los humedales como hábitat de aves acuáticas. Tópicos sobre humedales subtropicales y templados de Sudamérica. Oficina Regional de Ciencia y Tecnología de la UNESCO para América Latina y el Caribe-ORCYT-Montevideo-Uruguay, 219-228.

Borrini, G., Dudley, N., Jaeger, T., Lassen, B., Broome, N. P., Phillips, A., \& Sandwith, T. (2014). Gobernanza de áreas protegidas: de la comprensión a la acción. No. 20 de la Serie Directrices para buenas prácticas en áreas protegidas. Gland, Suiza: UICN. 10 p.

Cano, A., \& Díaz, F. (2002). Parques, áreas, Santuarios y reservas. Revista la Tadeo, (67), 177-182.

Castaño, U. (1999). Sierras y Serranías de Colombia. Banco de Occidente Credencial.

Castellanos, C. (2006). Los ecosistemas de humedales en Colombia. Revista Luna Azul. 1-5.

Castillo, F., Davidson, I., Duncan, C., Hahn, D., Luna, D., Mundkur, T., \& Vidal, R. (2011). Plan Estratégico Quinquenal, 2011-2015, de la Red Hemisférica de Reservas para Aves Playeras. Manomet Center for Conservation Sciences, Manomet, Massachusetts, EE.UU. 10 p.

CCA Comisión para la Cooperación Ambiental. (1999). Áreas Importantes para la Conservación de las Aves de América del Norte. Recuperado de: http://www3.cec.org/islandora/es/item/1664north-american-important-bird-areas-directory150-key-conservation-sites

Chaves M., \& Hurtado, A. (2007). Propuesta de un programa de investigación y monitoreo sobre prioridades temáticas del Sinap y mecanismos de articulación y cooperación interinstitucional para su implementación. Fondo para la Biodiversidad y las Áreas Protegidas y el Instituto de Investigación de Recursos Biológicos Alexander von Humboldt. Informe final. Bogotá D.C., Colombia. $99 \mathrm{p}$.

Conpes 3680. (2010). Lineamientos para la consolidación del Sistema Nacional de Áreas Protegidas. Ministerio de Ambiente, Vivienda y Desarrollo Territorial, Unidad Administrativa Especial del Sistema de Parques Nacionales Naturales. 38 p. 
Decreto 2372. (2010). Sistema Nacional de Áreas Protegidas, las categorías de manejo que lo conforman y se dictan otras disposiciones. Ministerio de Ambiente, Vivienda y Desarrollo Territorial. Colombia. $10 \mathrm{p}$.

De la Maza, J., Cadena, R., \& Piguerón, C. (2003). Estado actual de las áreas naturales protegidas de América Latina y el Caribe. México: PNUMA. 93 p.

De Groot, R., Stuip, M., Finlayson, M., \& Davidson, N. (2006). Valuing wetlands: guidance for valuing the benefits derived from wetland ecosystem services. Gland, Switzerland: International Water Management Institute. Recuperado de: https://www.ramsar.org/document/ramsar-technical-report-3-valuing-wetlands-guidance-forvaluing-the-benefits-derived-from

Duarte, S., Delgado, J., Gaviria, D., Goméz, L., \& Mejia, N. (2012). Atlas Parque Nacional Natural los Katíos. Parques Nacionales Naturales de Colombia. Santiago de Cali, Colombia: WWF. Recuperado de: http://awsassets.panda.org/downloads/katios_36_para_web_5.pdf

Díaz, J., \& Gómez, D. (2000). Programa nacional de investigación en biodiversidad marina y costera. Santa Marta: INVEMAR-FONADE-MMA. 83 p.

Díaz, J., Abadía, M., Arcadio, H., Robledo, C., \& Tejada, R. (2006). Parque Nacional Natural y sitio de Patrimonio Mundial los Katíos, plan de manejo. Unidad Administrativa Especial del Sistema de Parques Nacionales Naturales. Recuperado de: http://www.parquesnacionales.gov.co/portal/wpcontent/uploads/2013/12/PNNLosKatios.pdf

Franco, A., Devenish, C., Barrero, M., \& Romero, M. (2012). Colombia: Áreas importantes para la conservación de las aves América. Colombia. 135-148. En: Devenish, C., Díaz, D., Clay, R., Davidson, I., \& Yépez, I. (2009). Priority sites for biodiversity conservation. Eds. Important Bird Areas Americas. Quito, Ecuador: BirdLife International (BirdLife Conservation Series No. 16).

Fundación ProAves. (2014). El estado de las aves en Colombia 2014: Prioridades de conservación de avifauna colombiana. Conservación colombiana. 20 p.

Fundación ProAves. (2015). Aves de Colombia 2015 y estudios durante la filmación de Colombia magia salvaje. Conservación colombiana, (23) 1-49.

Fundación Humedales de Bogotá. (2018). Humedales Ramsar en Colombia. Recuperado de: http:// humedalesbogota.com/2018/01/25/humedalesramsar-colombia/
García, D. (2003). Planificación para la conservación y el uso sustentable de la biodiversidad en las reservas de la biósfera: avances en Colombia. En Memorias del V Congreso Interno, Instituto Alexander von Humboldt, Bogotá D.C., Colombia. $4 \mathrm{p}$.

García, C. (2010). Diagnóstico de las áreas marinas y costeras protegidas, y de las áreas de manejo en el Pacífico colombiano. Fundación MarViva, Colombia. Recuperado de: http://marviva.net/sites/default/files/documentos/amp_colombia.pdf

Garzón, N., \& Gutiérrez, J. (2013). Deterioro de humedales en el Magdalena medio: un llamado para su conservación. Fundación Alma, Instituto de Investigación de Recursos Biológicos Alexander von Humboldt. 145 p.

Gómez, I. (2011). Reserva de Biósfera el Tuparro: un reto para la conservación de la Orinoquía colombiana. Ambiente y Desarrollo, 15(29), 43-64.

Halffter, G. (2011). Reservas de la Biosfera: problemas y oportunidades en México. Acta zoológica mexicana, 27(1), 177-189.

Hansen, A., \& DeFries, R. (2007). Ecological mechanisms linking protected areas to surrounding lands. Ecological Applications, 17(4), 974-988.

Hernández, E. (2011). Programa de desarrollo de capacidades para el Caribe. Gestión del Patrimonio Natural. La Habana: Exclusivas Latinoamericanas ELA, S.L.

Herrón, P., Bessudo, S., \& Zambrano, H. (2007). Código de buenas prácticas para el Santuario de Fauna y Flora Malpelo. Parques Nacionales de Colombia y Fundación Malpelo.

Hurtado, A., Santamaría, M. \& Matallana, C. (2013). Plan de Investigación y Monitoreo del Sistema Nacional de Áreas Protegidas (SINAP): Avances construidos desde la Mesa de Investigación y Monitoreo entre 2009 y 2012. Instituto de Investigación de Recursos Biológicos Alexander von Humboldt y Parques Nacionales Naturales de Colombia. Bogotá, D. C., Colombia. 200 p.

IDEAM, IGAC, IAvH, Invemar, Sinchi \& IIAP. (2007). Ecosistemas continentales, costeros y marinos de Colombia. Instituto de Hidrología, Meteorología y Estudios Ambientales, Instituto Geográfico Agustín Codazzi, Instituto de Investigación de Recursos Biológicos Alexander von Humboldt, Instituto de Investigaciones Ambientales del Pacífico Jhon von Neumann, Instituto de Investigaciones Marinas y Costeras 
José Benito Vives De Andréis e Instituto Amazónico de Investigaciones Científicas Sinchi. Bogotá, D. C., Colombia: Imprenta Nacional de Colombia. 69 p.

Infante, O., Fuente, U., \& Atienza, J. (2011). Las Áreas Importantes para la Conservación de las Aves en España. SEO/BirdLife, Madrid.

Invemar. (2015). Informe del Estado de los Ambientes y Recursos Marinos y Costeros en Colombia. Recuperado de: http://www.invemar. org.co/documents/10182/14479/ier_2015_baja. pdf/f225fda1-eb06-44ad-9d60-535909e85cc6

López, J., \& Vidargas, F. (2016). Gestión y manejo del patrimonio mundial en México. Problemática, acciones y retos. Instituto Nacional de Antropología e Historia.

MADS Ministerio de Ambiente y Desarrollo Sostenible. (2012). Política nacional para la gestión integral de la biodiversidad y sus servicios ecosistémicos (PNGIBSE). Recuperado de: http://www.humboldt. org.co/images/pdf/PNGIBSE_español_web.pdf

MADS Ministerio de Ambiente y Desarrollo Sostenible. (2018). Lista de reservas de la biosfera. Recuperado de: http://www.minambiente.gov.co/index.php/ component/content/article/433-plantilla-bosquesbiodiversidad-y-servicios-ecosistematicos-26

Márquez, G. (2001). De la abundancia a la escasez: La transformación de ecosistemas en Colombia. (tesis doctoral). Universidad de los Andes en Mérida, Venezuela.

MEA Millennium Ecosystem Assessment. (2005). Ecosystems and Human Well-Being: Wetlands and Water Synthesis. World Resources Institute, Washington, D.C.

Ministerio de Medio Ambiente. (1996). Política Nacional de Biodiversidad. Departamento Nacional de Planeación, Instituto Alexander von Humboldt. Recuperado de: http://www.minambiente. gov.co/images/BosquesBiodiversidadyServiciosEcosistemicos/pdf/Politica-Nacional-de-Biodiversidad/politica_nacional-biodiversidad.pdf

Ministerio de Cultura. (2011). Patrimonio de la humanidad en Colombia. Bogota D.C., Colombia: Nomos Impresores. 19 p.

Mongabay (2016). The top 10 most biodiverse countries. What are the world's most biodiverse countries?. Recuperado de: https://news.mongabay. com/2016/05/top-10-biodiverse-countries/
Orellana, M. (2014). Tipología de instrumentos de derecho público ambiental internacional. Publicación de las Naciones Unidas, Santiago de Chile. Recuperado de: https://www.cepal.org/ es/publicaciones/37184-tipologia-instrumentosderecho-publico-ambiental-internacional

Pastrana, E., \& Pacheco, Y. (2010). La Convención Ramsar a lo largo del eje local-global: protección de humedales en el Valle del Cauca. Papel Político, 15(2), 573-616.

PNN Parques Nacionales Naturales. (2015). Plan de manejo del Santuario de Fauna y Flora Malpelo. Recuperado de: https://storage.googleapis.com/ pnn-web/uploads/2013/12/Plan-de-Manejo-SFFMalpelo_Adoptado_octubre-9_2015.pdf

Puerta, C., Hinestroza, P., \& Montoya, V. (2013). Cartografía sociocultural de Antioquia. Programa de Investigación Expedición Antioquia. 4 p.

Pulido, V. (2017). Conservación de las aves migratorias: rutas de migración en las Américas. Exégesis, 1(1), 33-43.

Ramsar. (2018). The List of Wetlands of International Importance. RAMSAR Secretariat: Gland, Switzerland. Recuperado de: http://biblioteca.cehum.org/bitstream/123456789/352/1/ SCR. $\% 20$ The $\% 20$ List $\% 20$ of $\% 20$ Wetlands $\% 20$ of $\% 20$ International\%20Importance.pdf

Renjifo, L., Franco, A., Álvarez, H., Álvarez, M., Borja, R., Botero, J., ..., \& Weber, W. (2000). Estrategia nacional para la conservación de las aves de Colombia. Instituto Alexander von Humboldt. Bogotá D.C., Colombia. 11 p.

Renjifo, L., Franco, A., Amaya, J., Kattán, G., \& López, B. (2002). Libro rojo de aves de Colombia. Serie Libros Rojos de Especies Amenazadas de Colombia. Instituto de Investigación de Recursos Biológicos Alexander von Humboldt, Ministerio del Medio Ambiente. Bogota D.C., Colombia. $50 \mathrm{p}$.

Ricaurte, L. (2014). Enfoque conceptual y metodológico para la identificación y monitoreo de humedales desde una perspectiva funcional y de servicios ecosistémicos. Instituto de Investigación de Recursos Biológicos Alexander von Humboldt. Bogotá, D.C., Colombia. 16 p.

Rodríguez, T. (2009). Convenios Internacionales y Ambiente: Recomendaciones para una mejor aplicación de los convenios en los países de la iniciativa del Corredor del Pacífico Este Trópica. San José de Costa Rica: Fundación Marviva. 32 p. 
Rodríguez, E., \& Giraldo, A. (2011). Características oceanográficas en isla Malpelo y su relación con la cuenca oceánica del Pacífico Colombiano. Boletín de Investigaciones Marinas y Costeras, (40), 19-32.

Romero M., Cabrera, E., \& Ortiz, N. (2008). Informe sobre el estado de la biodiversidad en Colombia 2006-2007. Instituto de Investigación Alexander von Humboldt. Bogotá, D.C., Colombia. 186 pp.

Romero, M. (2013). El sistema de áreas naturales protegidas y su rol para la conservación del agua. II Congreso Nacional de Derecho Agrario Provincial. La Plata, Argentina. Recuperado de: http://sedici.unlp.edu.ar/handle/10915/39557

Saldarriaga, L., Arango, D., Aristizábal, S., García, H., \& Arango, M. (2011). Cartilla vigías del patrimonio cultural Antioquia. Gobernación de Antioquia. Secretaría de Educación para la Cultura. Dirección de Fomento y Cultura. Medellin: Litoimpresos y servicios S.A. 34 p.

Schnack, J. (2001). Ecosistemas de Humedales. Aspectos históricos, conceptuales y de manejo. Recuperado de: http://sedici.unlp.edu.ar/bitstream/handle/10915/30731/Documento_completo.pdf?sequence $=1$

Secretaría de la Convención de Ramsar. (2016). Introducción a la Convención sobre los Humedales.
Gland, Suiza. Recuperado de: https://www.ramsar.org/sites/default/files/documents/library/ handbook1_5ed_introductiontoconvention_s_ final.pdf

SIAC Sistema de Información Ambiental de Colombia. (2018). Estrategias complementarias de conservación. Recuperado de: http://181.225.72.78/ Portal-SIAC-web/faces/Dashboard/Biodiversidad2/estrategias/estrategias.xhtml

UNESCO. (1972). Convención sobre la protección del Patrimonio Mundial, Cultural y Natural. Recuperado de: http://portal.unesco.org/es/ev.php-

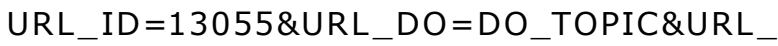
SECTION=201.html

UNESCO. (2018). Colombia, Properties inscribed on the World Heritage List. Recuperado de: http:// whc.unesco.org/en/statesparties/co/

Valencia, I. (2004). La convención de RAMSAR y las aves acuáticas en el neotrópico. Ornitologia neotropical, 15, 445-454.

Vargas, J. (2011). Restauración ecológica: biodiversidad y conservación. Acta Biológica Colombiana, 16(2), 221-246.

WHSRN Western Hemisphere Shorebird Reserve Network. (2018). Delta del Río Iscuandé. Recuperado de: https://www.whsrn.org/delta-delrio-iscuande. 
